\title{
Funciones inducidas e inducibles entre hiperespacios
}

\begin{abstract}
Alejandro Fuentes-Montes de Oca ${ }^{1}$
Resumen: En este artículo consideramos $\mathcal{H}(X)$ un hiperespacio de un continuo $X$. Sea $f: X \rightarrow Y$ una función continua entre continuos, se considera a la función inducida $\mathcal{H}(f): \mathcal{H}(X) \rightarrow \mathcal{H}(Y)$ dada por $\mathcal{H}(f)(A)=f(A)$, para todo $A \in \mathcal{H}(X)$. Por otro lado, si tenemos a la función continua $H: \mathcal{H}(X) \rightarrow \mathcal{H}(Y)$ y existe $g: X \rightarrow Y$ tal que $H=\mathcal{H}(f)$, decimos que $H$ es inducible. Se presentan tres clases de funciones entre continuos y se estudia el siguiente problema: $f$ pertenece a una clase si y sólo si la función inducida $\mathcal{H}(f)$ también pertenece a esa clase. Además, se presenta una caracterización para las funciones inducibles y con esto se muestra una aplicación a encajes ordenados.
\end{abstract}

Palabras clave: Continuo, hiperespacio, función inducida, función inducible y encaje ordenado.

\section{Induced and inducible mappings between hyperspaces}

\begin{abstract}
In this paper we consider $\mathcal{H}(X)$ be a hyperspace of a continuum $X$. Let $f: X \rightarrow Y$ be a continuous function between continua, consider the induced function $\mathcal{H}(f): \mathcal{H}(X) \rightarrow \mathcal{H}(Y)$ given by $\mathcal{H}(f)(A)=f(A)$, for all $A \in \mathcal{H}(X)$. On the other hand, if we have the continuous function $H: \mathcal{H}(X) \rightarrow \mathcal{H}(Y)$ and there exists $g: X \rightarrow Y$ such that $H=\mathcal{H}(f)$, we say that $H$ is inducible. Three classes of functions between continua are presented and the following problem is studied: $f$ belongs to a class if and only if the induced function $\mathcal{H}(f)$ also belongs to that class. In addition, a characterization for the inducible functions is presented and with this of sample an application to order embedding.
\end{abstract}

Keywords: Continuum, hyperspace, induced function, inducible function and order embedding.

Recibido: 12/06/2018. Aceptado: 20/11/2018. Publicado online: 31/12/2018. con revistapesquimat.matematica@unmsm.edu.pe

\footnotetext{
${ }^{1}$ UAEMéx, Facultad de Ciencias. e-mail: afuentesm@uaemex.mx
} 


\section{Introducción}

Este artículo está inspirado en los resultados presentados en [1], son dos los resultados de interés que se resuelven en dicho artículo. El primero es que dados dos espacios topológicos $X$ e $Y$, si $C(X)$ se puede encajar ordenadamente en $C(Y)$, entonces se puede encajar ordenadamente con un encaje inducible minimal. Y el segundo es que si $X$ es un continuo hereditariamente descomponible e $Y$ es un continuo indescomponible, entonces $C(X)$ no puede encajarse ordenadamente en $C(Y)$. La motivación es ver la posibilidad de hacer la generalización de dichos resultados a otros hiperespacios de continuos.

Complementamos la idea presentando algunos problemas de estudio en esta rama de la topología. Con esto nos referimos a lo siguiente: dada una función continua $f: X \rightarrow Y$ entre dos continuos $X$ e $Y$, se considera la función inducida $\mathcal{H}(f): \mathcal{H}(X) \rightarrow \mathcal{H}(Y)$ entre los hiperespacios $\mathcal{H}(X)$ y $\mathcal{H}(Y)$. Entonces nos preguntamos ¿si $f$ pertenece a una clase de funciones, entonces $\mathcal{H}(f)$ también pertenece, y viceversa? Por ejemplo, si la clase de funciones es la clase de los homeomorfismos, la pregunta sería if es un homeomorfismo si y sólo si $\mathcal{H}(f)$ es un homeomorfismo? Este problema no es nuevo y citar todos los trabajos al respecto nos llenaría de ellas, lo que hacemos es dar un panorama introductorio con tres clases de funciones para el hiperespacio $C_{n}(X)$, las cuales son estudiadas en [5].

El trabajo está dividido en cuatro partes, la primera es en la que nos encontramos. En la segunda parte presentamos las definiciones y los resultados generales que usamos en el desarrollo las partes siguientes. La tercera parte está dividida en tres subsecciones. En la subsección 3.1 se presenta el problema que acabamos de describir acerca de las funciones inducidas, presentando tres clases de funciones: abiertas, homeomorfismos y monótonas. En la sección 3.2 estudiamos condiciones necesarias y suficientes para que una función $H: \mathcal{H}(X) \rightarrow \mathcal{H}(Y)$ entre los hiperespacios $\mathcal{H}(X)$ y $\mathcal{H}(Y)$, sea inducible. En la sección 3.3 presentamos algunos resultados sobre encajes ordenados. En la cuarta parte damos las los comentarios finales del material que se presenta.

\section{Preliminares}

Iniciamos la sección escribiendo la definición del espacio en el que estamos interesados. Los trabajos en los que se estudia a este tipo de espacio topológico son bastantes, podemos referir al lector interesado en saber más a [10].

Definición 2.1 Un continuo es un espacio métrico, compacto y conexo. Un subconjunto de un continuo es un subcontinuo si el mismo es un continuo.

Definición 2.2 Sea $X$ un continuo. Si existen $A$ y $B$ subcontinuos propios de $X$ tales que $X=A \cup B$, entonces decimos que $X$ es descomponible. En caso de que no existan se le llama indescomponible. Además, decimos que $X$ es hereditariamente descomponible (indescomponible), si cada subcontinuo de $X$ es descomponible (indescomponible).

El siguiente resultado lo vamos a usar más adelante, una demostración la podemos encontrar en $[3$, Lema 12, p. 4].

Lema 2.3 Si $X$ es un continuo hereditariamente descomponible e $Y$ es un continuo indescomponible, entonces no existen funciones continuas de $X$ sobre $Y$.

Durante todo el trabajo llamaremos mapeo a una función continua.

Definición 2.4 Sea $f: X \rightarrow Y$ un mapeo entre continuos. 
1. $f$ es llamado abierto si para todo subconjunto abierto $A \subset X$, se cumple que $f(A)$ es abierto en $Y$.

2. Si $f$ es suprayectivo, entonces es llamado monótono siempre que para cada y $\in Y$ se tiene que $f^{-1}(y)$ es conexa en $X$.

3. $f$ es llamado un encaje si $X$ es homeomorfo a $f(X)$.

Se puede probar que si $f: X \rightarrow Y$ es monótono, entonces $f^{-1}(B)$ es conexa en $X$, para cada $B \subset Y$ cerrado y conexo. Ahora vamos a definir a los hiperespacios de un continuo, con los que vamos a trabajar.

Definición 2.5 Consideremos a un continuo $X$. Construyamos a las siguientes familias de subconjuntos de $X$ :

1. $2^{X}=\{A \subset X: A$ es cerrado no vacío $\}$ llamado el hiperespacio de cerrados;

2. para $n \in \mathbb{N}, C_{n}(X)=\left\{A \in 2^{X}: A\right.$ tiene a lo más $n$ componentes conexas $\}$ llamado el hiperespacio de a lo más n componentes;

3. $F_{1}(X)=\{\{x\}: x \in X\}$ llamado el hiperespacio de los unipuntuales.

Observación 2.6 Si d es la métrica asociada al continuo $X, a 2^{X}$ se le dota con una métrica llamada de Hausdorff inducida por la métrica d definida como:

$$
H_{d}(A, B)=\operatorname{máx}\{\sup \{d(a, B): a \in A\}, \sup \{d(b, A): b \in B\}\} .
$$

Para esto se puede consultar [9, Theorem (0.2), $p$. 2]. Con esto es fácil ver que tanto $F_{1}(X)$, como $C_{n}(X)$ son espacios métricos, para toda $n \in \mathbb{N}$. Más aún, todos estos hiperespacios son también continuos; para $2^{X}, C_{1}(X)$ y $F_{1}(X)$ consultar [9] y para $C_{n}(X)$ consultar [8, Theorem 3.1, p.240].

Observación 2.7 Podemos contruir una topología para $2^{X}$, llamada Topología de Vietoris, de la siguiente manera. Dada una colección finita $U_{1}, \ldots, U_{m}$ de conjuntos abiertos de $X$, definamos $\left\langle U_{1}, \ldots, U_{m}\right\rangle=\left\{A \in 2^{X}: A \subset \bigcup_{k=1}^{m} U_{k}\right.$ y $A \cap U_{k} \neq \emptyset$ para cada $\left.k \in\{1, \cdots, m\}\right\}$. La colección de los conjuntos de la forma $\left\langle U_{1}, \cdots, U_{m}\right\rangle$ forman una base para esta topología. Más aún, la topología inducida por la métrica de Hausdorff es la misma que la topología de Vietoris (ver [9, Theorem (0.13), p. 10]). Con esto podemos ver que $C_{n}(X)$ tiene una topología heredada de $2^{X}$, y para difrenciar usaremos la notación $\left\langle U_{1}, \ldots, U_{m}\right\rangle_{n}=C_{n}(X) \cap\left\langle U_{1}, \ldots, U_{m}\right\rangle$.

A lo largo del trabajo vamos a denotar a $C_{1}(X)$ como $C(X)$ y le llamaremos el hiperespacio de subcontinuos. Para un continuo $X$, el espacio $F_{1}(X)$ es homeomorfo a $X$, incluso son isométricos. Así, $F_{1}(X)$ es un subcontinuo de $C_{n}(X)$ para toda $n \in \mathbb{N}\left\{2^{X}\right\} \cup\left\{C_{n}(X): n \in \mathbb{N}\right\}$.

Definición 2.8 Sea $X$ un continuo. Un arco ordenado en $\mathcal{H}(X)$ es un arco $\alpha$ en $\mathcal{H}(X)$ tal que si $A, B \in \alpha$, entonces $A \subset B$ o $B \subset A$.

El siguiente resultado se encuentra en [6].

Proposición 2.9 Si $\alpha:[0,1] \rightarrow 2^{X}$ es un arco ordenado tal que $\alpha(0) \in C_{n}(X)$, entonces $\alpha \subset C_{n}(X)$. 
Definición 2.10 Sean $f_{1}, f_{2}: \mathcal{H}(X) \rightarrow \mathcal{H}(Y)$ mapeos entre hiperespacios. La relación $f_{1} \prec f_{2}$ si y sólo si $f_{1}(A) \subset f_{2}(A)$ para cada $A \in \mathcal{H}(X)$ es llamada relación de contención entre $f_{1}$ $y f_{2}$.

El siguiente resultado prueba que la relación de contención es una relación de orden.

Proposición 2.11 La relación de contención $\prec$ es una relación de orden en el conjunto de todos los mapeos entre $\mathcal{H}(X)$ y $\mathcal{H}(Y)$, este espacio se denota $C(\mathcal{H}(X), \mathcal{H}(Y))$.

Demostración. Tenemos que para $f: \mathcal{H}(X) \rightarrow \mathcal{H}(Y)$ se cumple que $f(A) \subset f(A)$, para todo $A \in \mathcal{H}(X)$. Así, $f \prec f$ y por lo tanto la relación es reflexiva. Sean $f_{1}, f_{2}: \mathcal{H}(X) \rightarrow \mathcal{H}(Y)$ tales que $f_{1} \prec f_{2}$ y $f_{2} \prec f_{1}$. Entonces $f_{1}(A) \subset f_{2}(A)$ y $f_{2}(A) \subset f_{1}(A)$, para todo $A \in \mathcal{H}(X)$. Así, $f_{1}=f_{2}$ y por lo tanto es antisimétrica. Sean $f_{1}, f_{2}, f_{3}: \mathcal{H}(X) \rightarrow \mathcal{H}(Y)$ tales que $f_{1} \prec f_{2}$ y $f_{2} \prec f_{3}$. Entonces $f_{1}(A) \subset f_{2}(A)$ y $f_{2}(A) \subset f_{3}(A)$, para todo $A \in \mathcal{H}(X)$. De esto se sigue que $f_{1} \prec f_{3}$ y por lo tanto es transitiva. Con todo esto tenemos que la relación de contención $\prec$ es una relación de orden.

\section{Funciones entre hiperespacios}

\subsection{Funciones inducidas}

A lo largo de esta parte vamos a trabajar con el siguiente concepto.

Definición 3.1 Sea un mapeo $f: X \rightarrow Y$, donde $X$ e $Y$ son dos continuos. A la función $\mathcal{H}(f): \mathcal{H}(X) \rightarrow \mathcal{H}(Y)$ definida por $\mathcal{H}(f)(A)=f(A)$ para todo $A \in \mathcal{H}(X)$, es llamada función inducida de $f$.

Cuando $\mathcal{H}(X)=2^{X}$, a $\mathcal{H}(f)$ se suele escribir como $2^{f}$. Tenemos que por [7, Lemma 13.3, p. 106] $2^{f}$ es un mapeo. Por lo tanto, cuando $\mathcal{H}(X)=C_{n}(X)$, para $n \in \mathbb{N}$, tenemos que $\mathcal{H}(f)$ es un mapeo. También tenemos que $\left.2^{f}\right|_{C_{n}(X)}=C_{n}(f)$.

Con todo esto tenemos el siguiente resultado, que muestra dos contenciones de conjuntos, la primera en el hiperespacio $\mathcal{H}(Y)$ y la segunda en el espacio $Y$.

Proposición 3.2 Sea $f: X \rightarrow Y$ un mapeo entre continuos y consideremos su mapeo inducido $\mathcal{H}(f)$. Asi tenemos que:

1. $\mathcal{H}(f)\left(F_{1}(X)\right) \subset F_{1}(Y)$;

2. si $A, B \in \mathcal{H}(X)$ tales que $A \subset B$, entonces $\mathcal{H}(f)(A) \subset \mathcal{H}(f)(B)$.

Demostración. Para la primera parte: sea $C \in \mathcal{H}(f)\left(F_{1}(X)\right)$, entonces existe $x_{1} \in X$ tal que $C=\mathcal{H}(f)\left(\left\{x_{1}\right\}\right)=f\left(\left\{x_{1}\right\}\right)=\left\{f\left(x_{1}\right)\right\} \in F_{1}(Y)$. Para la segunda parte: sea $y \in \mathcal{H}(f)(A)=$ $f(A)$, entonces existe $a \in A$ tal que $y=f(a)$. Por hipótesis $a \in B$, con lo que tenemos que $y=f(a) \in f(B)=\mathcal{H}(f)(B)$. Con todo esto hemos probado las dos contenciones.

Veamos ahora algunos resultados sobre ciertas familias de mapeos.

Teorema 3.3 Sea $f: X \rightarrow Y$ un mapeo entre continuos y $n \in \mathbb{N}$. Si $C_{n}(f): C_{n}(X) \rightarrow C_{n}(Y)$ es abierto, entonces $f$ es abierto.

Demostración. Sea $U$ un subconjunto abierto de $X$ y $x \in U$. Entonces $\langle U\rangle_{n}$ es un subconjunto abierto de $C_{n}(X)$. Por hipotesis tenemos que $C_{n}(f)\left(\langle U\rangle_{n}\right)$ es un abierto en $C_{n}(Y)$. Podemos verificar que $C_{n}(f)\left(\langle U\rangle_{n}\right) \subset\langle f(U)\rangle_{n}$. Dado que $\{f(x)\} \in C_{n}(f)\left(\langle U\rangle_{n}\right)$ existen subconjuntos 
abiertos $V_{1}, \ldots, V_{m}$ de $Y$ tales que $\{f(x)\} \in\left\langle V_{1}, \ldots, V_{m}\right\rangle_{n} \subset C_{n}(f)\left(\langle U\rangle_{n}\right) \subset\langle f(U)\rangle_{n}$. Con esto hemos probado que $f(x)$ es un punto interior de $f(U)$. Por lo tanto $f(U)$ es un subconjunto abierto de $Y$.

Observación 3.4 La implicación inversa del teorema anterior es falsa, para ver esto se puede consultar [5, Remark 9, p. 786].

Teorema 3.5 Sea $f: X \rightarrow Y$ un mapeo entre continuos y $n \in \mathbb{N}$. Si $f$ es un homeomorfismo, entonces $C_{n}(f): C_{n}(X) \rightarrow C_{n}(Y)$ es un homeomorfismo.

Demostración. Ya tenemos que $C_{n}(f)$ es continuo, entonces sólo hay que probar que es biyectivo y que es abierto.

Sean $A_{1}, A_{2} \in C_{n}(X)$. Supongamos que $C_{n}(f)\left(A_{1}\right)=C_{n}(f)\left(A_{2}\right)$, entonces $f\left(A_{1}\right)=f\left(A_{2}\right)$. Como $f$ es un homeomorfismo se da que $A_{1}=A_{2}$. Por otro lado, si $B \in C_{n}(Y)$, entonces es fácil comprobar que $f^{-1}(B) \in C_{n}(X)$. Por lo tanto $C_{n}(f)\left(f^{-1}(B)\right)=B$. Con todo esto hemos probado que $C_{n}(f)$ es biyectiva.

Sea $\mathcal{C}$ un abierto en $C_{n}(X)$. Probemos que $C_{n}(f)(\mathcal{C})$ es abierto en $C_{n}(Y)$. Sea $B \in C_{n}(f)(\mathcal{C})$. Consideremos $A \in C_{n}(X)$ tal que $C_{n}(f)(A)=B$. Como $\mathcal{C}$ un abierto, entonces existen subconjuntos abiertos $U_{1}, \ldots, U_{m}$ de $X$ tales que $A \in\left\langle U_{1}, \ldots, U_{m}\right\rangle_{n} \subset \mathcal{C}$. Así

$$
B \in\left\langle C_{n}(f)\left(U_{1}\right), \ldots, C_{n}(f)\left(U_{m}\right)\right\rangle_{n}=\left\langle f\left(U_{1}\right), \ldots, f\left(U_{m}\right)\right\rangle_{n} \subset C_{n}(f)(\mathcal{C}) .
$$

Como $f$ es abierto, se tiene que $B$ es un punto interior de $C_{n}(f)(\mathcal{C})$. Por lo tanto $C_{n}(f)(\mathcal{C})$ es un subconjunto abierto de $C_{n}(Y)$, en consecuencia $C_{n}(f)$ es abierto. Finalmente podemos concluir que $C_{n}(f)$ es un homeomorfismo.

Observación 3.6 La implicación inversa del teorema anterior es verdadera, para ver esto se puede consultar [5, Theorem 46, p. 801].

Teorema 3.7 Sea $f: X \rightarrow Y$ un mapeo entre continuos y $n \in \mathbb{N}$. Entonces $f$ es monótono si y sólo si $C_{n}(f): C_{n}(X) \rightarrow C_{n}(Y)$ también lo es.

Demostración. Supongamos primero que $f$ es monótono. Sea $B \in C_{n}(Y)$. Por la hipotesis tenemos que $f^{-1}(B)$ tiene el mismo número de componentes que $B$. Sea $A \in\left(C_{n}(f)\right)^{-1}(B)$. Dado que $C_{n}(f)(A)=f(A)=B$ y $A \subset f^{-1}(B)$ (ver [5, Lemma 2, p. 784]), tenemos que $A$ intersecta a cada componente de $f^{-1}(B)$. Por la Proposición ?? y por [9, Theorem (0.8), p. 59] existe un arco ordenado $\alpha:[0,1] \rightarrow C_{n}(X)$ tal que $\alpha(0)=A$ y $\alpha(1)=f^{-1}(B)$. Dado que $B=$ $f(A) \subset f(\alpha(t)) \subset f\left(f^{-1}(B)\right)=B$ para cada $t \in[0,1]$, concluimos que $\alpha([0,1]) \subset\left(C_{n}(f)\right)^{-1}(B)$. Con esto hemos probado que $\left(C_{n}(f)\right)^{-1}(B)$ es arco conexa y por lo tanto conexa, lo que se deseaba.

Supongamos ahora que $f$ no es monótono. Al suponer esto, existe $y \in Y$ tal que $f^{-1}(y)$ no es conexo. Entonces existen dos subconjuntos cerrados disjuntos $K$ y $L$ de $X$ tales que $f^{-1}(y)=$ $K \cup L$. Sean $\mathcal{A}=\left\{A \in\left(C_{n}(f)\right)^{-1}(\{y\}): A \subset K\right\}$ y $\mathcal{B}=\left\{A \in\left(C_{n}(f)\right)^{-1}(\{y\}): A \cap L \neq \emptyset\right\}$. Entonces $\mathcal{A}$ y $\mathcal{B}$ son subconjuntos cerrados disjuntos no vacíos de $C_{n}(X)$ tales que $\left(C_{n}(f)\right)^{-1}(\{y\})=$ $\mathcal{A} \cup \mathcal{B}$. Así, $C_{n}(f)$ no es monótono.

\subsection{Funciones inducibles}

A lo largo de esta parte vamos a trabajar con el concepto de la siguiente definición. Podemos notar ahora que al revés de las funciones inducidas, se trata de hallar una función entre los continuos dada una función entre hiperespacios. 
Definición 3.8 Sean $X$ e $Y$ continuos y sea $g: \mathcal{H}(X) \rightarrow \mathcal{H}(Y)$. Decimos que $g$ es inducible siempre que exista un mapeo $f: X \rightarrow Y$ tal que $g=\mathcal{H}(f)$.

El siguiente teorema es probado en [4] para cuando $\mathcal{H}(X)=2^{X}$ y $\mathcal{H}(X)=C(X)$. Para cuando $\mathcal{H}(X)=C_{n}(X)$ el resultado es mencionado en [5]. Aquí damos la prueba para cualquier $\mathcal{H}(X)$ haciendo unos cuantos sencillos ajustes.

Teorema 3.9 Sean $X$ e $Y$ continuos y sea $g: \mathcal{H}(X) \rightarrow \mathcal{H}(Y)$ un mapeo entre hiperespacios. Tenemos que $g$ es inducible si y sólo si cada una de las siguientes condiciones se cumplen:

1. $g\left(F_{1}(X)\right) \subset F_{1}(Y)$;

2. $A \subset B$ implica que $g(A) \subset g(B)$ para todo $A, B \in \mathcal{H}(X)$;

3. $g$ es minimal respecto al orden $\prec$.

Demostración. Probemos primero la necesidad. Supongamos que $g$ es inducible, esto es, existe $f: X \rightarrow Y$ tal que $g=\mathcal{H}(f)$. Por la Proposición 3.2 tenemos que (1) y (2) se cumplen.

Probemos (3). Supongamos que existe $g_{0}$ tal que cumple (2) y $g_{0} \prec \mathcal{H}(f)$. Entonces para cada $x \in X$ tenemos $g_{0}(\{x\}) \subset \mathcal{H}(f)(\{x\})=\{f(x)\}$ y por tanto $g_{0}(\{x\})=\{f(x)\}$. Para cada $A \in \mathcal{H}(f)$ y cada $x \in A$, de donde por $(2) g_{0}(\{x\}) \subset g_{0}(A)$. Si tomamos la unión sobre todos los puntos de $A$ tenemos $\bigcup\left\{g_{0}(\{x\}): x \in A\right\}=\bigcup\{\{f(x)\}: x \in A\}=f(A) \subset g_{0}(A)$. Como esto se cumple para todo $A \in \mathcal{H}(f)$, se concluye que $\mathcal{H}(f) \prec g_{0}$. Por la Proposición 2.11 $\mathcal{H}(f)=g_{0}$ y con esto terminamos la prueba de (3).

Probemos ahora la suficiencia. Supongamos que $g: \mathcal{H}(X) \rightarrow \mathcal{H}(Y)$ cumple (1), (2) y (3). Definamos $f: X \rightarrow Y$ poniendo $f(x)$ como el único punto de $g(\{x\})$. Entonces para cada $A \in \mathcal{H}(X)$ tenemos $\mathcal{H}(f)(A)=f(A)=\bigcup\{\{f(x)\}: x \in A\}=\bigcup\{g(\{x\}): x \in A\} \subset g(A)$, esto por $(2)$. Hemos probado que $\mathcal{H}(f) \prec g$ y por $(3) \mathcal{H}(f)=g$, lo cual indica que $g$ es inducible.

Revisamos algunos ejemplos de funciones que no son inducibles, para ver los detalles que no se prueban se puede consultar [4].

Ejemplo 3.10 Sean $I=[0,1], A \in \mathcal{H}(I)$ y $D(A)$ el diametro de A. Definamos la bola cerrada de radio $r$ como $B(A, r)=\{x \in I$ : existe $y \in A$ tal que $|x-y| \leq r\}$. Sea $g: \mathcal{H}(I) \rightarrow \mathcal{H}(I)$ dada por $g(A)=B(A, D(A))$. Tenemos que $g(\{x\})=\{x\}$, asi que (1) del Teorema 3.9 se cumple. Sean $A, B \in \mathcal{H}(X)$ tales que $A \subset B$. Se puede probar sin mucha complicación que $g(A) \subset g(B)$, asi que (2) también se cumple. Pero resulta que (3) no se va a cumplir, y para esto consideremos la identidad id $: \mathcal{H}(I) \rightarrow \mathcal{H}(I)$. Como $A \subset B(A, D(A))$, se cumple que id $(A) \subset g(A)$. Por lo tanto id $\prec g$, pero $i d \neq g$. Con todo esto vemos que $g$ no es inducible.

Ejemplo 3.11 Sea $g: \mathcal{H}(I) \rightarrow \mathcal{H}(I)$ dada por $g(A)=\{$ mín $A\}$. Es claro que si $A \subset B$, no necesariamente mín $A=$ mín $B$. Por tanto (2) no se cumple $y$ en consecuencia $g$ no es inducible. Tenemos que (1) y (3) si se cumplen.

Ejemplo 3.12 Sean $X=I$ e $Y$ un arco de pseudo-arcos. Para la contrucción del continuo hereditariamente indescomponible llamado pseudo-arco se puede consultar [10, Exercise 1.23, p. 13]. Sea $f: Y \rightarrow X$ la proyección natural (el mapeo cociente). Consideremos $g: C(X) \rightarrow C(Y)$ dada por $g(A)=f^{-1}(A)$. Como $f$ es un mapeo abierto, tenemos que $g$ es continua. Para ver que (1) no se cumple, si $\{x\} \in F_{1}(X)$, entonces $g(\{x\})$ es un pseudo-arco. Por lo tanto, $g$ no es inducible. Tenemos que (2) y (3) si se cumplen. 


\subsection{Encajes ordenados}

En esta sección trataremos un problema bastante estudiado en la topología, este es cuándo un espacio está contenido dentro de otro espacio preservando su estructura. La siguiente definición explica el concepto.

Definición 3.13 Sean $X$ e $Y$ dos continuos. Decimos que el hiperespacio $\mathcal{H}(X)$ se puede encajar ordenadamente en el hiperespacio $\mathcal{H}(Y)$ siempre que exista un encaje $H: \mathcal{H}(X) \rightarrow \mathcal{H}(Y)$ tal que si $A, B \in \mathcal{H}(X)$ y $A \subset B$, entonces $H(A) \subset H(B)$.

Proposición 3.14 Si $h: X \rightarrow Y$ es un encaje entre continuos, entonces $\mathcal{H}(h): \mathcal{H}(X) \rightarrow \mathcal{H}(Y)$ es un encaje ordenado.

Demostración. Por hipotesis tenemos que $h: X \rightarrow h(X)$ es un homeomorfismo, así por el Teorema 3.5 tenemos que $\mathcal{H}(h): \mathcal{H}(X) \rightarrow \mathcal{H}(h)(\mathcal{H}(X))$ es un homeomorfismo. Por la Proposición $3.2 \mathcal{H}(h)$ es ordenado. Por lo tanto $\mathcal{H}(h)$ es un encaje ordenado.

Ejemplo 3.15 Consideremos al continuo $S^{1}$ como el círculo unitario en $\mathbb{R}^{2}$. Sabemos por [7, Examples 5.1 and 5.2, p.p. 33 and 35] que $C(I)$ y $C\left(S^{1}\right)$ son homeomorfos a una 2-celda $I \times I$. Si consideramos a $I \times I$ como una variedad, tenemos que $I$ es un elemento de la frontera de la variedad $C(I)$ y $S^{1}$ no es un elemento de la frontera de la variedad $C\left(S^{1}\right)$. Con esto podemos probar que $C\left(S^{1}\right)$ se puede encajar en $C(I)$, pero no se puede encajar ordenadamente. Por otro lado, como I si se puede encajar en $S^{1}$, entonces por la Proposición $3.14 C(I)$ se puede encajar ordenadamente en $C\left(S^{1}\right)$.

El siguiente resultado muestra que si $\mathcal{H}(X)$ puede ser encajado ordenadamente en $\mathcal{H}(Y)$, entonces existe un encaje ordenado minimal. Pero no sólo eso, además prueba que ese encaje será inducible. Dando con esto una relación entre los encajes ordenados y las funciones inducibles, en los hiperespacios de continuos.

Teorema 3.16 Sean $X$ e $Y$ dos continuos. Si $H: \mathcal{H}(X) \rightarrow \mathcal{H}(Y)$ es un encaje ordenado tal que $H\left(F_{1}(X)\right) \subset F_{1}(Y)$, entonces existe un encaje ordenado inducible minimal $G_{0}: \mathcal{H}(X) \rightarrow \mathcal{H}(Y)$.

Demostración. Vamos a usar la relación de contención $\prec$ en $C(\mathcal{H}(X), \mathcal{H}(Y))$, que denota el espacio de los mapeos de $\mathcal{H}(X)$ en $\mathcal{H}(Y)$. Consideremos la siguiente colección de cerrados en $C(\mathcal{H}(X), \mathcal{H}(Y))$

$$
\mathcal{F}=\{G \in C(\mathcal{H}(X), \mathcal{H}(Y)): G \text { es un encaje ordenado y } G \prec H\} .
$$

Es claro que $H \in \mathcal{F}$ y en consecuencia $\mathcal{F} \neq \emptyset$. Sea $\left\{G_{n}\right\}_{n \in \mathbb{N}}$ una sucesión decreciente en $\mathcal{F}$. Sea $G^{\prime}: \mathcal{H}(X) \rightarrow \mathcal{H}(Y)$ dada por $G^{\prime}(A)=\bigcap_{n \in \mathbb{N}} G_{n}(A)$. Como $G_{1}(A) \supset G_{2}(A) \supset G_{3}(A) \supset \ldots$ tenemos que $G^{\prime}(A) \in \mathcal{H}(Y)$, esto quiere decir que $G^{\prime}$ está bien definida. Si $A_{1} \subset A_{2}$, entonces $G^{\prime}\left(A_{1}\right)=\bigcap_{n \in \mathbb{N}} G_{n}\left(A_{1}\right) \subset \bigcap_{n \in \mathbb{N}} G_{n}\left(A_{2}\right)=G^{\prime}\left(A_{2}\right)$. Además, $G^{\prime} \prec G_{1} \prec H$. Por lo tanto $G^{\prime} \in \mathcal{F}$. Por el Teorema de Reducción de Brouwer (consultar [11, Theorem 11.1, p.17]) aplicado a la colección $\mathcal{F}$, tenemos que tiene un elemento minimal $G_{0}$. Observemos que todo elemento de $\mathcal{F}$ cumple (2) y (3) del Teorema 3.9, y dado que $G_{0}\left(F_{1}(X)\right) \subset H\left(F_{1}(X)\right) \subset F_{1}(Y)$ se cumple (1). Por lo tanto $G_{0}$ es un encaje ordenado inducible minimal.

Como una aplicación a la proposición anterior tenemos el siguiente resultado.

Teorema 3.17 Sea $X$ un continuo hereditariamente descomponible. Si $Y$ es un continuo indescomponible, entonces no existen encajes ordenados de $\mathcal{H}(X)$ en $\mathcal{H}(Y)$ tal que $H\left(F_{1}(X)\right) \subset$ $F_{1}(Y)$. 
Demostración. Supongamos que si existe $F: \mathcal{H}(X) \rightarrow \mathcal{H}(Y)$ un encaje ordenado. Por el teorema anterior existe $G_{0}: \mathcal{H}(X) \rightarrow \mathcal{H}(Y)$ inducible minimal. Entonces existe un mapeo $g: X \rightarrow Y$ tal que $\mathcal{H}(g)=G_{0}$, lo cual no es posible por el Lema 2.3. Por lo tanto no existen encajes ordenados de $\mathcal{H}(X)$ en $\mathcal{H}(Y)$.

\section{Comentarios finales}

Si consideramos a $\mathfrak{M}$ una clase de funciones, por ejemplo las que estudiamos en este artículo: abiertas, homeomorfismos y monótonas. Los problemas de ver bajo que condiciones $f \in \mathfrak{M}$, implica que $\mathcal{H}(f) \in \mathfrak{M}$ y bajo que condiciones $\mathcal{H}(f) \in \mathfrak{M}$, implica que $f \in \mathfrak{M}$, ya han sido bastante estudiados. Se puede consultar la tabla [7, p. 381] que reúne los resultados para ciertas clases de funciones para $2^{X}$ y $C(X)$. Para $C_{n}(X)$ se puede consultar [5]. A pesar de que hay varios trabajos que estudian estos problemas, hay todavía muchas cosas por contestar y para darse una idea se puede consultar [2].

Con respecto a las funciones inducibles, hay un teorema más general que el Teorema 3.9. Dicho teorema aparece en [2, Theorem 5.2, p. 256] y agrega a los hiperespacios que presentamos otros hiperespacios, estos son $C_{\infty}(X), F_{n}(X)(n \in \mathbb{N})$ y $F_{\infty}(X)$. El lector interesado podrá ver que la demostración es casi idéntica a la que se presenta aquí.

Finalmente, dejamos las siguientes preguntas: Sea $\mathcal{H}(f) \in\left\{C_{\infty}(X), F_{n}(X), F_{\infty}(X)\right\}$ con $n \in \mathbb{N}$,

1. ¿Si $X$ e $Y$ dos continuos tales que $\mathcal{H}(X)$ puede encajerse ordenadamente en $\mathcal{H}(Y)$, entonces existe un encaje ordenado inducible minimal de $\mathcal{H}(X)$ en $\mathcal{H}(Y)$ ?

2. ¿Si $X$ un continuo hereditariamente descomponible y si $Y$ es un continuo indescomponible, entonces no existen encajes ordenados de $\mathcal{H}(X)$ en $\mathcal{H}(Y)$ ?

\section{Referencias bibliográficas}

[1] Aliaga, A., Chupayo, H., Contreras, P., Olano, W., Rubio, M., Villegas, L (2016). Encajes ordenados inducibles entre hiperespacios. Pesquimat, XIX(2), 9-13.

[2] Charatonik, J. J. (2003). Recent Research in Hyperspace Theory. Extracta Mathematicae, 18(2), 235-262.

[3] Charatonik, W. J. (2010). Propiedades que se preservan bajo funciones confluentes. IV Taller de continuos e hiperespacios. Morelia, Michoacan, México.

[4] Charatonik, J. J., Charatonik, W. J. (1998). Inducible mappings between hyperspaces. Bulletin of the Polish, 46(1), 5-9.

[5] Charatonik, J. J., Illanes, A., Macías, S. (2002). Induced mappings on some hyperspaces $C_{n}(X)$ of a continuum $X$. Houston Journal of Mathematics, 28(4), 781-805.

[6] Hosokawa, H. (2011). Srong size levels of $C_{n}(X)$. Houston Journal of Mathematics, 37(2), 955-965.

[7] Illanes, A., Nadler Jr., S. B. (1999). Hyperspaces, Fundamentals and Recent Advances. Monographs and Textbooks in Pure and Applied Mathematics. New York, USA: Marcel Dekker, Inc. 
[8] Macías, S. (2001). On the hyperspaces $C n(X)$ of a continuum $X$. Topology and its Applications, $109(2), 237-256$

[9] Nadler Jr., S. B. (1978). Hyperspaces of sets . Monographs and Textbooks in Pure and Applied Mathematics. New York, USA: Marcel Dekker, Inc.

[10] Nadler Jr., S. B. (1992). Continuum theory: An introduction. Monographs and Textbooks in Pure and Applied Mathematics. New York, USA: Marcel Dekker, Inc.

[11] Whyburn, G. T. (1971). Analytic Topology. Vol. 28. United States of America: American Mathematical Society 